\title{
Comparison of angiotensin II type 1-receptor blockers to regress pressure overload-induced cardiac hypertrophy in mice
}

\begin{abstract}
Lei $\mathrm{Li}^{1,2,4}$, Ning Zhou ${ }^{1,4}$, Hui Gong ${ }^{2}$, Jian $\mathrm{Wu}^{1,2}$, Li Lin ${ }^{1}$, Issei Komuro ${ }^{3}$, Junbo Ge ${ }^{1,2}$ and Yunzeng Zou ${ }^{1,2}$
Angiotensin II (AngII) type 1-receptor blockers (ARBs) have been effectively used not only in the treatment of hypertension but also in cardiac protection. However, whether and why there are differences in these effects still remain unclear. Here we compared the effects of five commonly used ARBs (Candesartan, Olmesartan, Losartan, Telmisartan and Valsartan) on pressure overload-induced cardiac hypertrophy in mice model. Pressure overload was produced by constriction of the transverse aorta (TAC) for 2 weeks, which induced a significant elevation of blood pressure; ARBs or saline was administered through a stomach tube; Cardiac hypertrophy was evaluated by transthoracic echocardiography, cardiac histology and specific gene expression analyses. Although all the five ARBs, which did not repress the elevation of left ventricular pressure after TAC, attenuated the development of cardiac hypertrophy in the wild-type mice, the degrees of regression by Candesartan, Olmesartan and Losartan tended to be larger than those by Telmisartan and Valsartan. Furthermore, in angiotensinogen-knockout mice lacking endogenous AngII, TAC-induced cardiac hypertrophy was regressed by Candesartan, Olmesartan and Losartan but not by Telmisartan and Valsartan administration. Our data suggest that Candesartan, Olmesartan and Losartan can effectively inhibit pressure overloadinduced cardiac hypertrophy even in the absence of Angll, whereas Telmisartan and Valsartan could exert the inhibitory effects only in the presence of Angll.
\end{abstract}

Hypertension Research (2010) 33, 1289-1297; doi:10.1038/hr.2010.182; published online 14 October 2010

Keywords: Angiotensin II; Angiotensin II type 1-receptor blockers; cardiac hypertrophy; pressure overload

\section{INTRODUCTION}

Cardiac hypertrophy is not only a physiologically adaptive state before heart failure, but also an independent risk factor of major cardiac events. ${ }^{1}$ Although there are many factors that can induce cardiac hypertrophy, hypertensive stimulation is a major hypertrophy-inducing factor. ${ }^{2,3}$ Angiotensin II (AngII) and its type 1-receptor (AT1-R) have been known to be greatly involved in pressure overload-induced cardiac hypertrophy. ${ }^{4,5}$ AT1-R blockers (ARBs) are clinically available as a highly effective and well-tolerated class of drugs for the treatment of hypertension. In addition, a variety of clinical evidences have shown that ARBs provide cardiovascular protection beyond blood pressure (BP) lowering. ${ }^{6}$ Especially, ARB administration effectively prevents cardiac hypertrophy and improves the cardiovascular outcomes in patients with hypertension. ${ }^{6,7}$ At present, there are several ARBs widely used in clinics, such as Losartan, Irbesartan, Telmisartan, Candesartan, Valsartan, Olmesartan and so on. Although some of them have been shown to have more beneficial effects than other types of antihyperten- sive agents such as $\beta$-adrenergic receptor blockers and calcium channel blockers, there are also reports indicating that cardiovascular outcomes by ARB-based treatment do not differ from those by other agent-based treatment. ${ }^{8,9}$ We therefore supposed whether there were differences in cardiac protection among ARBs besides antihypertensive potency.

AT1-R is a member of the $G$ protein-coupled receptor superfamily ${ }^{10}$ and ARBs are highly selective in binding to the AT1-R and blocking diverse effects mediated by AT1-R. ${ }^{6,11,12}$ Structurally, ARBs not only have the common biphenyl-tetrazole ring but also the unique side chains, which contribute to drug-specific differences in pharmacokinetic and pharmacodynamic properties and antihypertensive potency. ${ }^{6,13}$ Many in vitro studies including ours have suggested that the effects of ARBs on suppression of AT1-R activation differ. ${ }^{11,12,14}$ However, a rigorous in vivo comparison of different ARBs to regress pressure overload-induced cardiac hypertrophy has not been performed. Moreover, we have recently reported that Candesartan inhibited activation of AT1-R and attenuated cardiac hypertrophy induced

\footnotetext{
${ }^{1}$ Central Laboratory, Shanghai Institute of Cardiovascular Diseases, Zhongshan Hospital, Fudan University, Shanghai, China; ${ }^{2}$ Research Center for Cardiovascular Diseases, Institutes of Biomedical Sciences, Fudan University, Shanghai, China and ${ }^{3}$ Department of Cardiovascular Science and Medicine, Chiba University Graduate School of Medicine, Chiba, Japan

${ }^{4}$ These authors contributed equally to this work.

Correspondence: Professor J Ge or Professor Y Zou, Institutes of Biomedical Sciences, Shanghai Institute of Cardiovascular Diseases, Zhongshan Hospital, Fudan University, 180 Feng Lin Road, Shanghai 200032, China.

E-mail: jbge@zs-hospital.sh.cn or zou.yunzeng@zs-hospital.sh.cn

Received 6 May 2010; revised 9 July 2010; accepted 13 July 2010; published online 14 October 2010
} 
by pressure overload in the absence of AngII ${ }^{11}$ however, it is unknown whether this holds true for other ARBs.

In this study, we compared the potencies of five commonly used ARBs, including Losartan, Candesartan, Telmisartan, Valsartan and Olmesartan, in the regression of pressure overload-induced cardiac hypertrophy in the presence or absence of endogenous AngII by using wild type (WT) and angiotensinogen-knockout (ATG KO) mice, respectively, and found that Candesartan, Olmesartan and Losartan can effectively inhibit pressure overload-induced cardiac hypertrophy even in the absence of Ang II, whereas Telmisartan and Valsartan could exert the effect only in the presence of AngII.

\section{METHODS}

\section{Animals and pressure overload model}

Male WT (C57/BL6J, commercially purchased from Shanghai Laboratory Animal Center, Chinese Academy, Shanghai, China) and ATG KO mice (kindly provided by Issei Komuro, Chiba University, Japan), aged 10 weeks, were used in the present study. Generation of the ATG KO mice has been previously described. ${ }^{15}$ There were no significant differences in body weight, heart weight, heart weight to body weight ratio (heart weight/body weight), BP and heart rate between the two types of mice at basal state. Pressure overload was produced by constriction of transverse aorta (TAC) for 2 weeks as described previously. ${ }^{16,17}$ Briefly, mice were anesthetized by intraperitoneal injection of a cocktail of ketamine $\left(100 \mathrm{mg} \mathrm{kg}^{-1}\right)$ and xylazine $\left(5 \mathrm{mg} \mathrm{kg}^{-1}\right)$, and respiration was artificially controlled with a tidal volume of $0.2 \mathrm{ml}$ and a respiratory rate of 110 breaths $\mathrm{min}^{-1}$. The TAC was constricted with 7-0 nylon strings by ligating the aorta with a blunted 27-gauge needle to yield a narrowing of $0.4 \mathrm{~mm}$ in diameter when the needle was pulled out later. All of the animal experiments were performed in compliance with the Guide for the Care and Use of Laboratory Animals published by the US National Institutes of Health (NIH Publication No. 85-23, revised 1996) and was approved by the guidelines of Fudan University.

\section{Oral administration of ARBs}

All ARBs including Losartan (Merck Pharmaceutical, Hangzhou, China), Candesartan (Takeda Pharmaceutical, Tianjin, China), Telmisartan (Boehringer Ingelheim Pharmaceutical, Shanghai, China), Valsartan (Novartis Pharmaceutical, Beijing, China) and Olmesartan (Daiichi Sankyo Pharmaceutical, Shanghai, China) were purchased commercially. WT and ATG KO mice were randomly divided into seven groups for each genotype of mice: saline plus sham-operated mice (sham, $n=5$ for WT, $n=4$ for ATG KO), saline plus TAC group (saline, $n=9$ for WT, $n=8$ for $A T G \mathrm{KO}$ ), Candesartan plus TAC group (Candesartan, $n=10$ for WT, $n=8$ for ATG KO), Olmesartan plus TAC group (Olmesartan, $n=10$ for WT, $n=9$ for $A T G \mathrm{KO}$ ), Losartan plus TAC group (Losartan, $n=8$ for WT, $n=8$ for $A T G \mathrm{KO}$ ), Telmisartan plus TAC group (Telmisartan, $n=10$ for WT, $n=8$ for $A T G \mathrm{KO}$ ) and Valsartan plus TAC group (Valsartan, $n=9$ for WT, $n=8$ for $A T G \mathrm{KO}$ ). ARBs were dissolved in saline, and equal volumes of freshly prepared ARB solution or saline $(0.2 \mathrm{ml})$ were administered to mice once daily through an oral gavage starting from 3 days before the operation to the end of the experiments. The dosages for Candesartan, Olmesartan, Losartan, Telmisartan and Valsartan administration were 2.1, 5.4, 13.4, 21.4 and $21.4 \mathrm{mg} \mathrm{kg}^{-1}$ day $^{-1}$, respectively. Selection of drug dosages was based upon Bios formula, clinically relevant concentrations and our preliminary study.

\section{Echocardiography and hemodynamic measurements}

Transthoracic echocardiography was performed before and at 2 weeks after TAC or sham operation using a $30-\mathrm{MHz}$ high-frequency scanhead (VisualSonics Vevo770, VisualSonics, Toronto, ON, Canada). ${ }^{17}$ All measurements were averaged for three consecutive cardiac cycles and were carried out by three experienced technicians who were unaware of the identities of the respective experimental groups. Left ventricle (LV) hemodynamics was also evaluated before and at the end of study. Briefly, a micronanometer catheter (Millar 1.4F, SPR 835, Millar Instruments, Houston, TX, USA) was inserted through the right common carotid artery into the aorta and carefully introduced into LV.
The transducer was connected to a Power Laboratory system (AD Instruments, Castle Hill, Australia), and BP, heart rate, LV end-systolic pressure (LVESP) and LV end-diastolic pressure (LVEDP) were measured.

\section{Morphology and histological analysis}

Mice were killed and hearts were excised. For global morphometry, hearts were perfused with PBS followed by $4 \%$ polyformaldehyde. For histological analysis, heart tissues were fixed in 10\% formalin and embedded in paraffin or frozen in liquid nitrogen, sectioned at $4-\mu \mathrm{m}$ thickness, and stained with hematoxylin and eosin or Masson trichrome, respectively. Digital photographs were taken at magnification $\times 200$ or $\times 400$, respectively. For measurement, five random highpower fields from each section were chosen and quantified in a blinded manner. The extent of fibrosis was expressed as the ratio of Masson trichrome-stained area to total LV wall area. The cross-sectional area of cardiac myocytes and midtransverse sectional area of the hearts were analyzed quantitatively by morphometry of hematoxylin and eosin-stained sections. Five sections for each heart were counted. The original images were measured by using an automated image analysis system (Image-Pro Plus 5.0, Media Cybernetics, Bethesda, MD, USA).

\section{Analysis of hypertrophic gene expression}

Total RNA was prepared from the heart tissues of mice by using TRIzol reagent (15596-018, Gibco BRL, Carlsbad, CA, USA); the reverse transcription PCR was performed by using TOYOBO ReverTra Ace- $\alpha$-RT-PCR kit according to the manufacturer's instruction. The real-time PCR was performed on a BioRad IQ5 multicolor detection system by using $2 \mu \mathrm{g}$ of synthesized cDNA. Atrial natriuretic peptide (ANP), skeletal a-actin (SAA), sarcoplasmic reticulum $\mathrm{Ca}^{2+}$ ATPase (SERCA2) and glyceraldehyde-3-phosphate dehydrogenase were amplified using their specific primers. The primers used for PCR analysis are as follows: for ANP gene, 5'-ACGCAGCTTGGTCACATTGC-3' and 5'-CCACTAGAC CACTCATCTAC-3'; for SAA gene, 5'-AGCAGATGTGGATCACCAAG-3' and 5'-CTGCAACCACAGCACGATTG-3'; for SERCA2 gene, 5'-GAAGCAGTAGA CAGATGTTGG-3' and 5'-GCTGTGAGAAGCTGTGAGCA-3'; for glyceraldehyde-3-phosphate dehydrogenase gene, 5'-CCACTCTTCCACCTTCGATG-3' and $5^{\prime}$-TCCACCACCCTGTTGCTGTA-3'. A comparative $C_{\mathrm{T}}$ method was used to determine the relative quantification of RNA expression, which involves comparing the $C_{\mathrm{T}}$ values of the samples of interest with a control or calibrator such as a non-treated sample or RNA from normal tissue. All real-time PCR reactions were performed in triplicate.

\section{Statistical analysis}

Data are expressed as mean \pm s.e.m. Multiple-group comparison was performed by one-way analysis of varience followed by least-significant difference procedure for comparison of means. Comparison between two groups under identical conditions was performed by the two-tailed Student's $t$-test. A value of $P<0.05$ was considered statistically significant.

\section{RESULTS}

\section{Elevation of aortic blood pressure (ABP) and LV pressures} after TAC

Literally, the inhibition of the cardiac hypertrophy of each ARB is associated with their dosages administrated. In the present study, however, we compared the efficacy of each ARB beyond their antihypertensive effects. Thus, we try to determine the maximal dosage of each ARB without anti-hypertensive effects. We treated the TAC mice with four different dosages of each ARB and examined the ABP of the mice so as to draw a dose-ABP curve (shown in Supplementary Figure). We found that dosages higher than 2.1, 5.4, 13.4, 21.4 and $21.4 \mathrm{mg} \mathrm{kg}^{-1} \mathrm{day}^{-1}$ of Candesartan, Olmesartan, Losartan, Telmisartan and Valsartan, respectively, will lower the ABP of mice to some extent and therefore lead to different after-loading of the mice in each group. Different pressure loads will lead to different hypertrophic responses. To observe the anti-hypertrophy effects of these five ARBs beyond their lowering blood pressure, we used the dosages of each ARB to guarantee an equal-effective dose for a fair comparison. 
We also confirmed changes of hemodynamic parameters (LVESP and LVEDP) after TAC and observed the effects of ARBs administration on them. LVESP and LVEDP were measured in mice after 2 weeks of TAC. There were no significant differences in hemodynamic parameters between the two genotypes of mice at basal state (data not shown). TAC for 2 weeks induced a significant elevation of LVESP (from $80-110$ to $150-180 \mathrm{~mm} \mathrm{Hg}$ ) compared with sham operation (Figure 1a), which was similar between the WT and the ATG KO mice, and ARB administration did not suppress the elevation of LVESP (Figure 1b). LVEDP remained unchanged among all groups (Figure 1c). These results indicated that TAC produced a similar pressure overload in the WT and ATG KO mice, which could not be repressed by $\mathrm{ARB}$ treatment.

\section{Effects of five ARBs on cardiac hypertrophy as analyzed by} echocardiography

Cardiac hypertrophy was first evaluated by echocardiography (Figure 2a). Quantitative analysis revealed that both the LV endsystolic posterior wall thickness (LVPWs) and LV end-diastolic posterior wall thickness (LVPWd) were significantly increased by 2 weeks of TAC but not by sham operation, and the increases in LVPWs and LVPWd by TAC were similar between the WT and the ATG KO mice (Figure 2b). On treatment with the five ARBs, all significantly inhibited increment of the LV wall thickness by TAC in the WT mice (Figure 2b). Although they were not significantly different, the degrees of inhibition by Candesartan, Olmesartan and Losartan tended to be larger compared with those by Telmisartan and Valsartan in the WT mice (Figure 2b). In the ATG KO mice, however, treatment with Candesartan, Olmesartan and Losartan significantly attenuated the increases in LVPWs and LVPWd by TAC, whereas Telmisartan and Valsartan administration could not do so. On the other hand, the LV internal dimension at end systole and diastole (LVIDs and LVIDd) had no changes among all the groups (Figure 2c), and the LV ejection fraction (LVEF) also remained unchanged in all enrolled animals
(Figure 2d). These results suggested that although all the five ARBs could effectively inhibit pressure overload-induced increases in LV wall thickness in the presence of AngII, Candesartan, Olmesartan and Losartan could exert this effect even in the absence of AngII, and that ARB administration did not affect cardiac functions in the early phase of TAC.

Effects of ARBs on morphological changes evaluated by histological analysis

After 2 weeks of TAC, the hearts of mice were globally enlarged (Figure 3a), and heart weight/body weight ratio was significantly increased in the mice of saline-treated groups (Figure 3b). The midtransverse section area of LV wall (Figures $4 \mathrm{a}$ and c) and the crosssectional area of individual cardiac myocytes (Figures $4 \mathrm{~b}$ and d) were also significantly increased after TAC in saline-treated mice. These changes by TAC were similar between the WT and the ATG KO mice. However, among the ARB-treated groups, the changes of morphological parameters were quite different. The increase either in heart size and weight (Figures $3 \mathrm{a}$ and $\mathrm{b}$ ) or in individual cardiomyocyte size (Figures $4 \mathrm{a}-\mathrm{d}$ ) induced by TAC was significantly abolished by all five ARBs in the WT mice, whereas in the ATG $\mathrm{KO}$ mice the inhibitory effects were only observed in Candesartan-, Olmesartan- and Losartan-treated groups but not in Telmisartan- and Valsartan-treated mice.

\section{Effects of ARBs on myocardial fibrosis induced by TAC}

Myocardial fibrosis is increased in the development of cardiac hypertrophy, which accounts for the formation of cardiac remodeling. ${ }^{18,19}$ We therefore examined the extent of fibrosis in myocardium by histological staining after 2 weeks of TAC. The extent of fibrosis in LV wall was significantly increased after TAC in all saline-administered mice (Figures $5 \mathrm{a}$ and $\mathrm{b}$ ). The increase was significantly suppressed by all the five ARBs in the WT mice (Figures $5 \mathrm{a}$ and $\mathrm{b}$ ). In the ATG KO mice, however, although the extent of LV fibrosis was

\section{a}

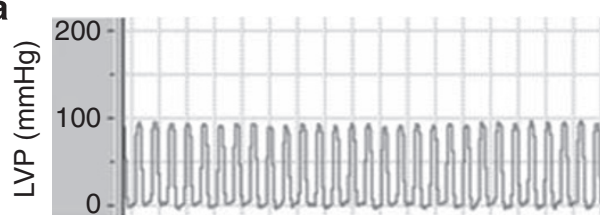

sham



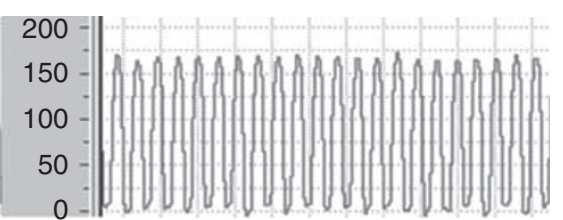

TAC

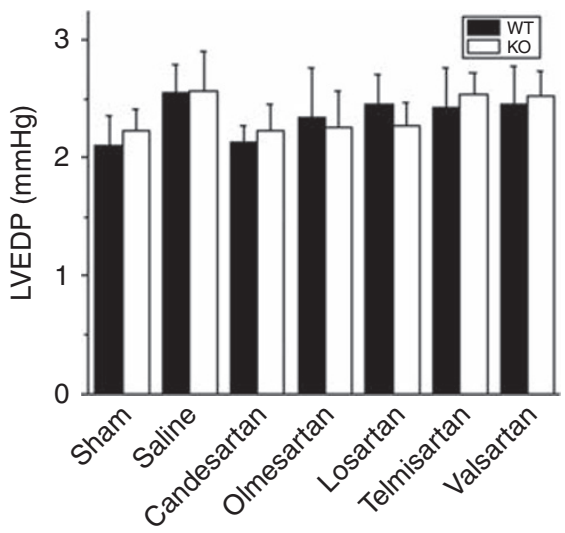

Figure 1 Hemodynamic parameters of mice subjected to TAC and treated with saline or ARBs for 2 weeks. (a) Representative recordings of LV pressures from sham- or TAC-operated and saline-treated WT mice. (b) Quantitative analyses for LVESP and LVEDP. Data are expressed as mean \pm s.e.m. from 4-10 mice. ${ }^{*} P<0.05$ vs. sham-operated mice of the same genotype. Candesartan, Olmesartan, Losartan, Telmisartan and Valsartan: Candesartan, Olmesartan, Losartan, Telmisartan or Valsartan plus TAC, respectively; KO, ATG KO mice; Saline, TAC+saline; Sham, sham+saline; WT, wild-type mice. 


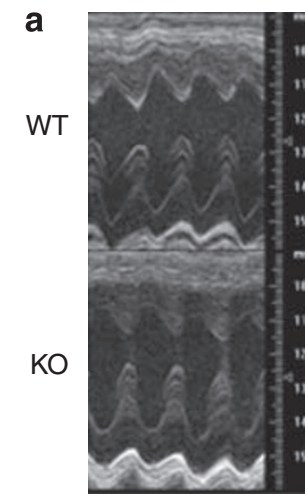

Sham

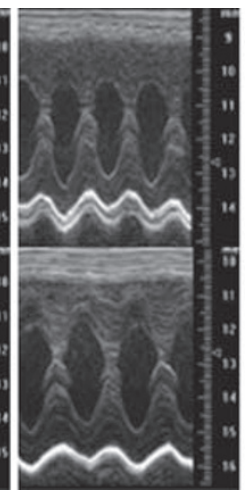

Saline

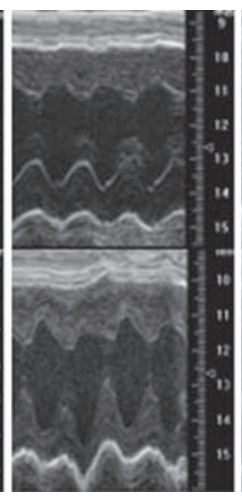

Candesartan

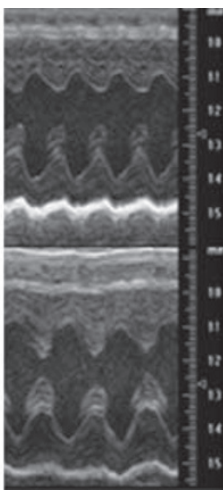

Olmesartan

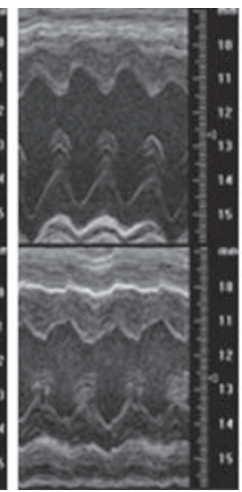

Losartan

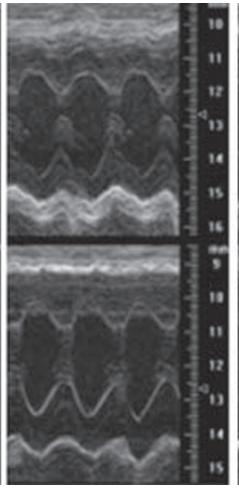

Telmisartan

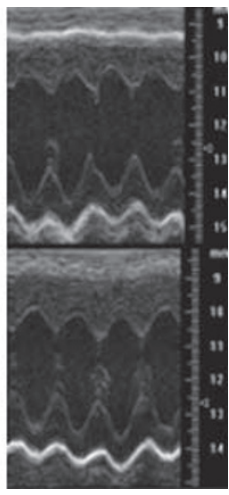

Valsartan
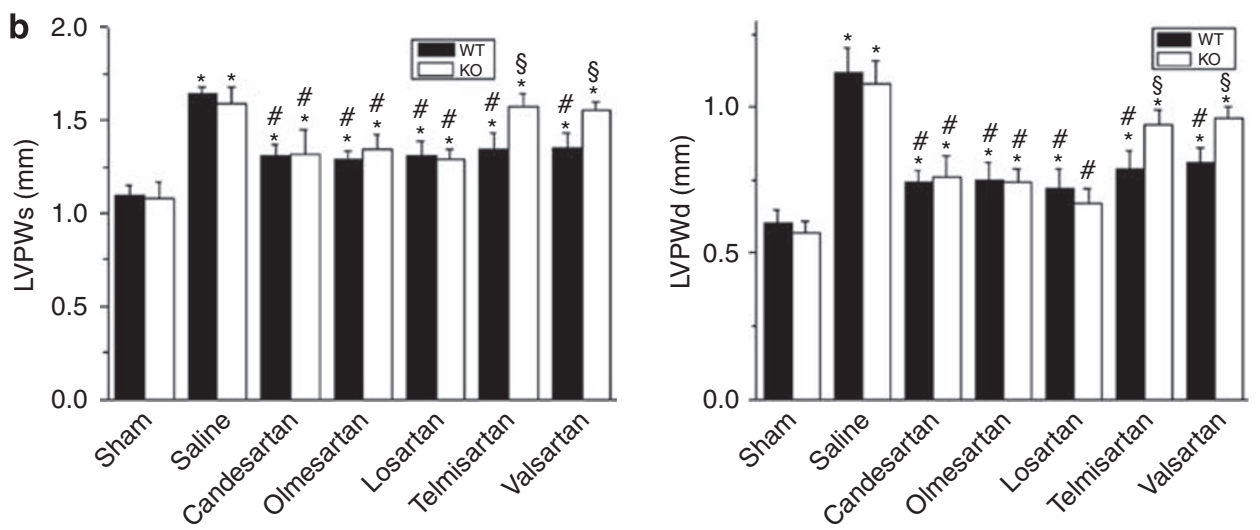

C
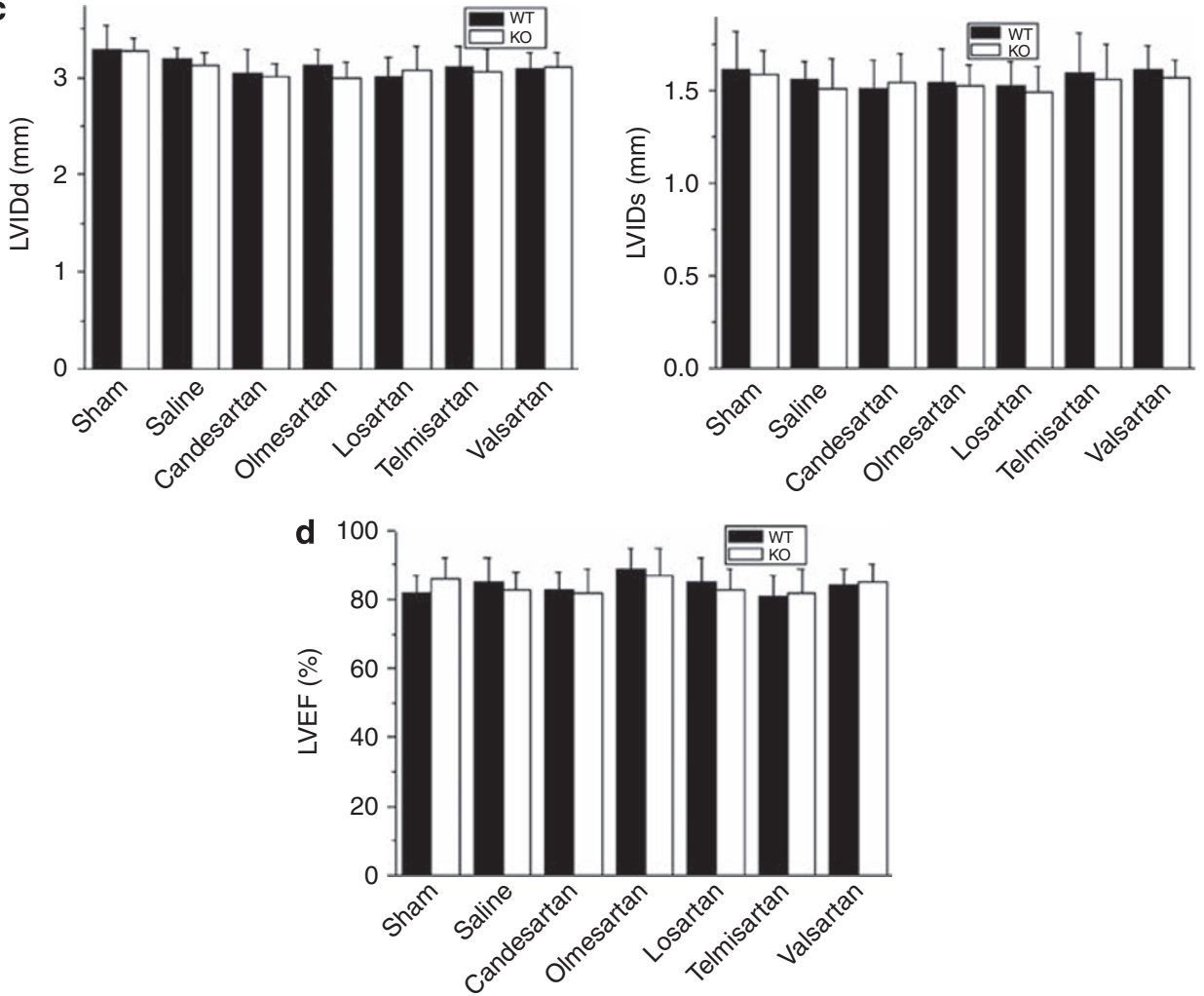

Figure 2 Echocardiographic analysis. (a) Representative M-mode tracings are shown. (b-d) Quantifications of LVPWs, LVPWd, LVIDs, LVIDd and LVEF. ${ }^{*} P<0.05$ vs. sham-operated mice of the same genotype; ${ }^{\#} P<0.05$ vs. saline-treated TAC mice of the same genotype; ${ }^{\S} P<0.05$ vs. same ARB-treated WT mice with TAC. 


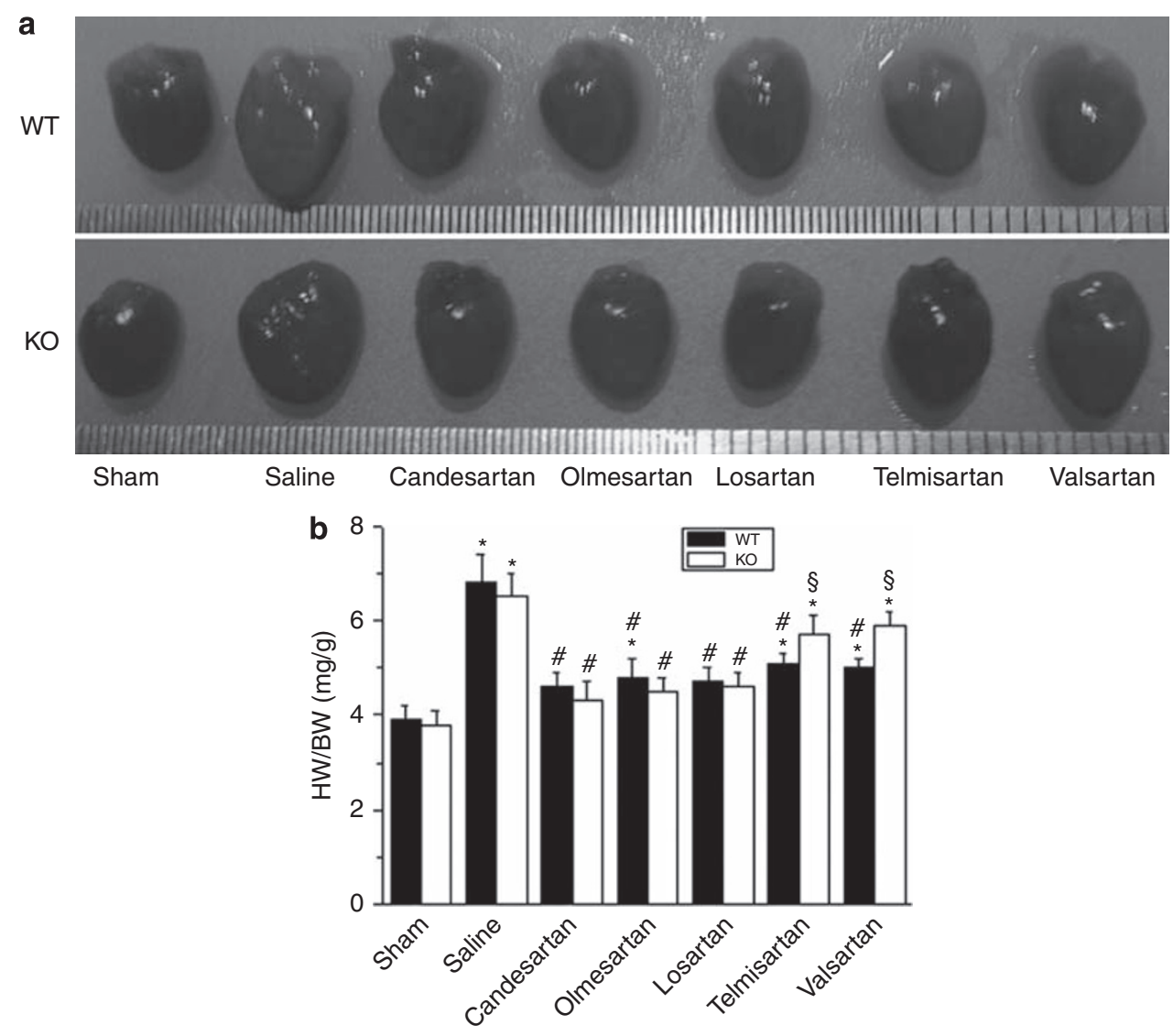

Figure 3 Morphological analyses for the hearts. (a) Representative photographs of whole hearts. (b) Quantifications of heart weight/body weight (HW/BW). ${ }^{*} P<0.05$ vs. sham-operated mice of the same genotype; ${ }^{\#} P<0.05$ vs. saline-treated TAC mice of the same genotype; ${ }^{\circledR} P<0.05$ vs. same ARB-treated WT mice with TAC.

similarly increased as in the WT mice by TAC, the increase in LV fibrosis by TAC was significantly suppressed in the mice of the Candesartan, Olmesartan and Losartan groups but not in those of the Telmisartan and Valsartan groups (Figures $5 \mathrm{a}$ and $\mathrm{b}$ ).

Effects of ARBs on hypertrophic gene expression induced by TAC Reprogramming of specific gene expression is one of the hypertrophic responses to hemodynamic overload. ${ }^{20,21}$ We thus examined the expressions of ANP, SAA and SERCA2 genes in the hearts at 2 weeks after TAC. TAC significantly upregulated the mRNA levels of ANP and SAA genes and downregulated the expression of SERCA2 gene compared with sham operation, and the upregulation in ANP and $S A A$ and the downregulation of SERCA2 gene expression were significantly suppressed in all ARB groups of the WT mice (Figures $6 \mathrm{a}-\mathrm{c})$. In the $A T G \mathrm{KO}$ mice, however, TAC for 2 weeks induced a similar pattern of reprogramming of the three genes as in the WT mice (Figures $6 \mathrm{a}-\mathrm{c}$ ), but the inhibitory effects on the TAC-induced gene reprogramming were significantly different among the five ARBs. Although Candesartan, Olmesartan and Losartan administration might abrogate the reprogramming of $A N P, S A A$ and SERCA2 gene expression, the reprogramming of gene expression could not be reversed by treatment with Telmisartan and Valsartan (Figures 6a-c).

\section{DISCUSSION}

This study demonstrated that the effects of different ARBs on cardiac protection are quite diverse. Under a similar hypertensive condition, Candesartan, Olmesartan and Losartan abrogated not only AngII- but also mechanical stress-induced cardiac hypertrophy, whereas Telmisartan and Valsartan only suppressed AngII-related hypertrophic responses.

Although ARBs share a common mode of action that blocks AngIImediated responses, the antihypertensive potency differs according to the drugs. ${ }^{6,13}$ In this study, under a similarly hypertensive stimulation, although the antihypertrophic effects were not significantly different among the five ARBs, the degrees of regression of cardiac hypertrophy by Candesartan, Olmesartan and Losartan seemed to be larger than those by Telmisartan and Valsartan. Especially in the absence of endogenous AngII, the regression of cardiac hypertrophy by Candesartan, Olmesartan and Losartan was more significant than that by Telmisartan and Valsartan, indicating that the antihypertrophic efficacy also differs according to the drugs. We have previously indicated that besides agonist-dependent activation, AT1-R can be activated directly by mechanical stress independently of AngII. ${ }^{11}$ It is therefore considerable that in the WT mice, TAC can induce cardiac hypertrophy at least by both AngII and mechanical stress stimuli. Although all the five ARBs antagonize AngII-mediated responses, only some of them, including Candesartan, Olmesartan and Losartan, can inhibit mechanical stress-induced activation of AT1-R after TAC. In the ATG KO mice, however, as TAC induces cardiac hypertrophy independently of AngII, only those possessing the ability to inhibit mechanical stressinduced AT1-R activation could function after TAC.

Although the reason for the differences in the effects on cardiac protection among ARBs is not clearly explained, the pharmacokinetics such as bioavailability, duration of half-life and route of elimination 


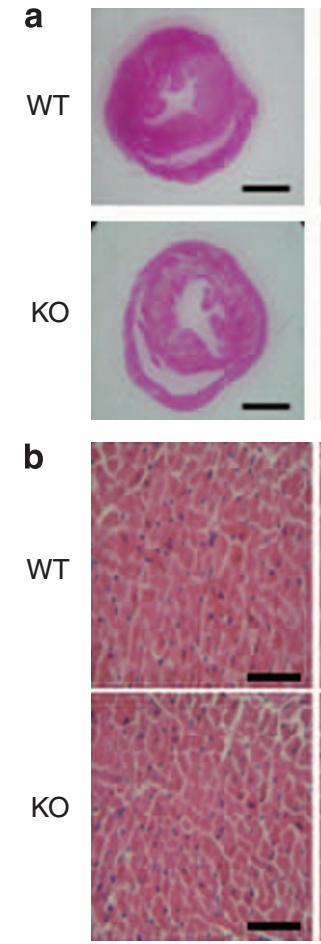

Sham
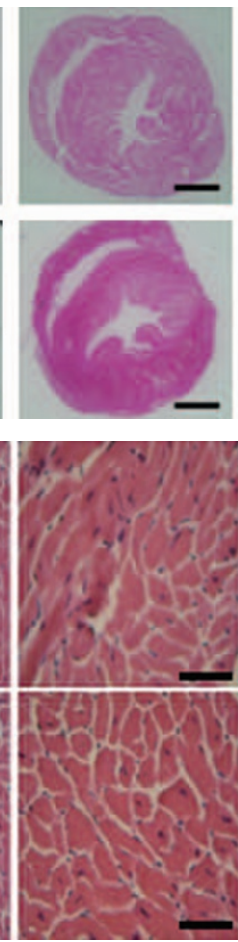

Saline


Candesartan
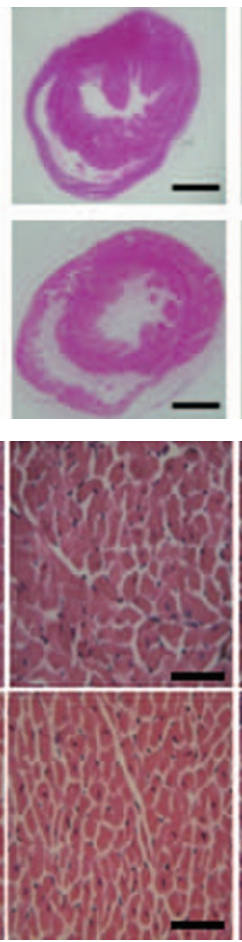

Olmesartan
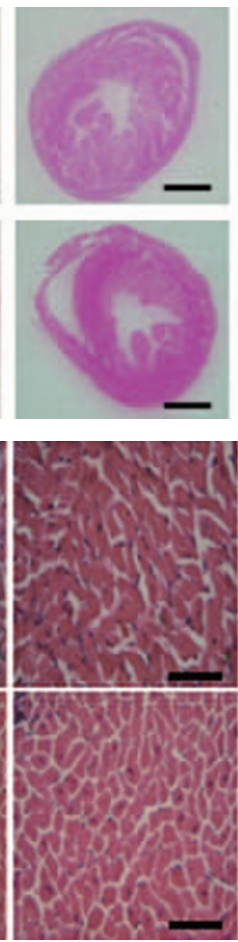

Losartan
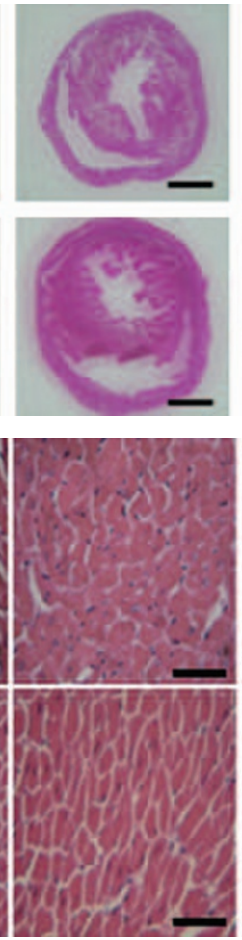

Telmisartan
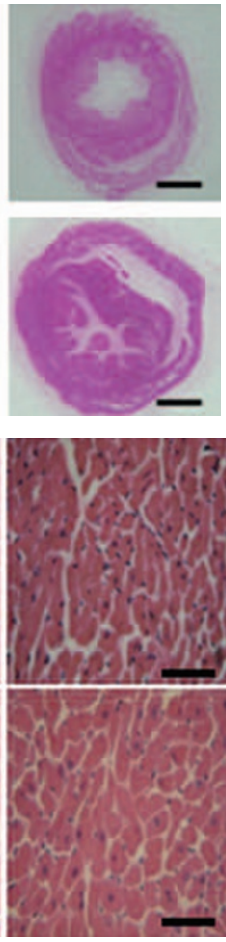

Valsartan
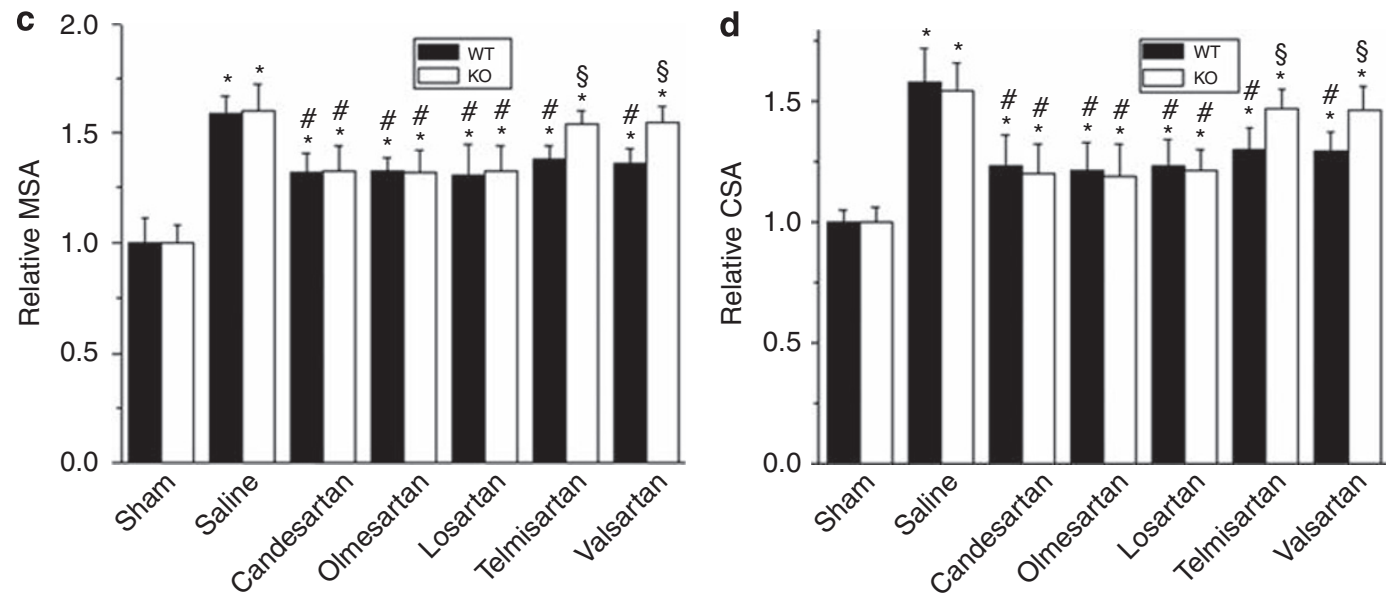

Figure 4 Histological analyses for the hearts. (a) Representative photographs of hematoxylin and eosin-stained heart sections (scale bar: $2 \mathrm{~mm}$ ). (b) Highermagnification views of hematoxylin and eosin staining (scale bar: $30 \mu \mathrm{m}$ ). (c) Quantitative analysis of mid-transverse section area (MSA). (d) Quantitative analysis of the cross-sectional area (CSA) of cardiac myocytes. ${ }^{*} P<0.05$ vs. sham-operated mice of the same genotype; ${ }^{\#} P<0.05$ vs. saline-treated TAC mice of the same genotype; ${ }^{\S} P<0.05$ vs. same ARB-treated WT mice with TAC.

and the chemical structures are considerable. ${ }^{6,13}$ The different efficacies of ARBs may be based on the differences in the chemical structures, which determine their unique pharmacological properties. Insurmountable antagonism is one of the pharmacological properties that are relevant to the efficacies of ARBs. Insurmountable behavior reflects tight binding and slow dissociation of the drug and receptor, and insurmountable ARBs can maximally suppress the AngII-induced responses. ${ }^{22}$ Olmesartan and Candesartan have been shown to have an in vitro insurmountability against AngII-induced AT1-R activation. ${ }^{11,12,23}$ Recently, inverse agonism against AT1-R has attracted great attention. An inverse agonist stabilizes inactive conformation of the receptor and reduces constitutive activity of the receptor or the agonist-independent receptor activity. ${ }^{24,25}$ It has been indicated by in vitro studies that both Candesartan and Olmesartan strongly inhibit AngII-independent, mechanical stretch-induced activation, as well as the constitutive activity of the AT1-R, ${ }^{11,12}$ suggesting that Candesartan and Olmesartan may act as an inverse agonist against AT1-R. This study provided an in vivo evidence for the insurmountability and inverse agonism of Candesartan and Olmesartan against AT1-R. With regard to Losartan, it has been indicated that neither the constitutive activity of AT1-R nor the mechanical stretch-induced activation of the receptor in cultured cells could be inhibited by Losartan, suggesting that Losartan itself cannot act as an in vitro inverse agonist of the AT1-R. However, EXP3174, the active metabolite of Losartan, can inhibit constitutive activity of the AT1-R. ${ }^{11}$ As Losartan exerts it's effect mainly through EXP3174 in the body, we 


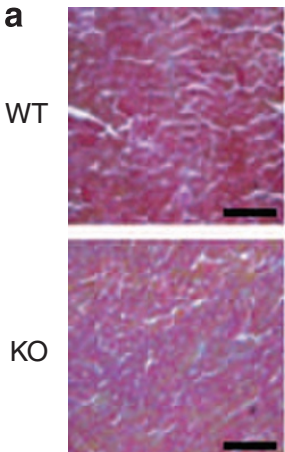

Sham

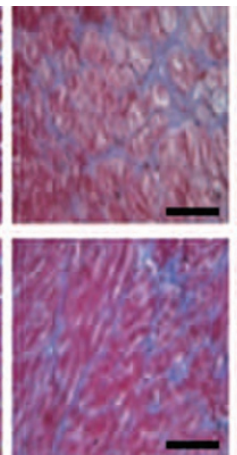

Saline
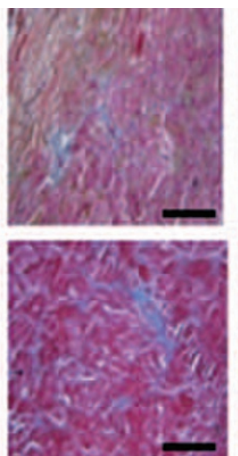

Candesartan
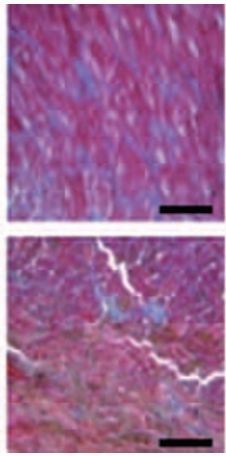

Olmesartan
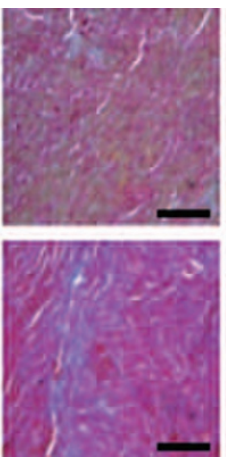

Losartan
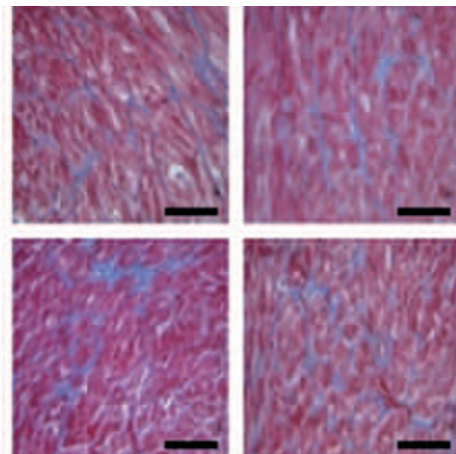

Telmisartan

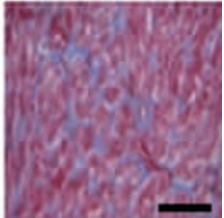

Valsartan

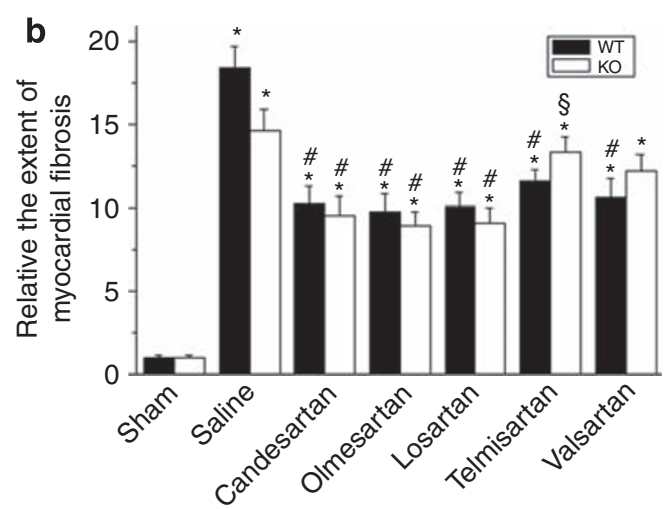

Figure 5 Myocardial fibrosis. (a) Representative photographs of Masson-trichrome staining in LV sections (scale bar: $30 \mu \mathrm{m}$ ). (b) Quantitative analyses for the extent of myocardial fibrosis. ${ }^{*} P<0.05$ vs. sham-operated mice of the same genotype; ${ }^{\#} P<0.05$ vs. saline-treated TAC mice of the same genotype; ${ }^{\S} P<0.05$ vs. same ARB-treated WT mice with TAC.
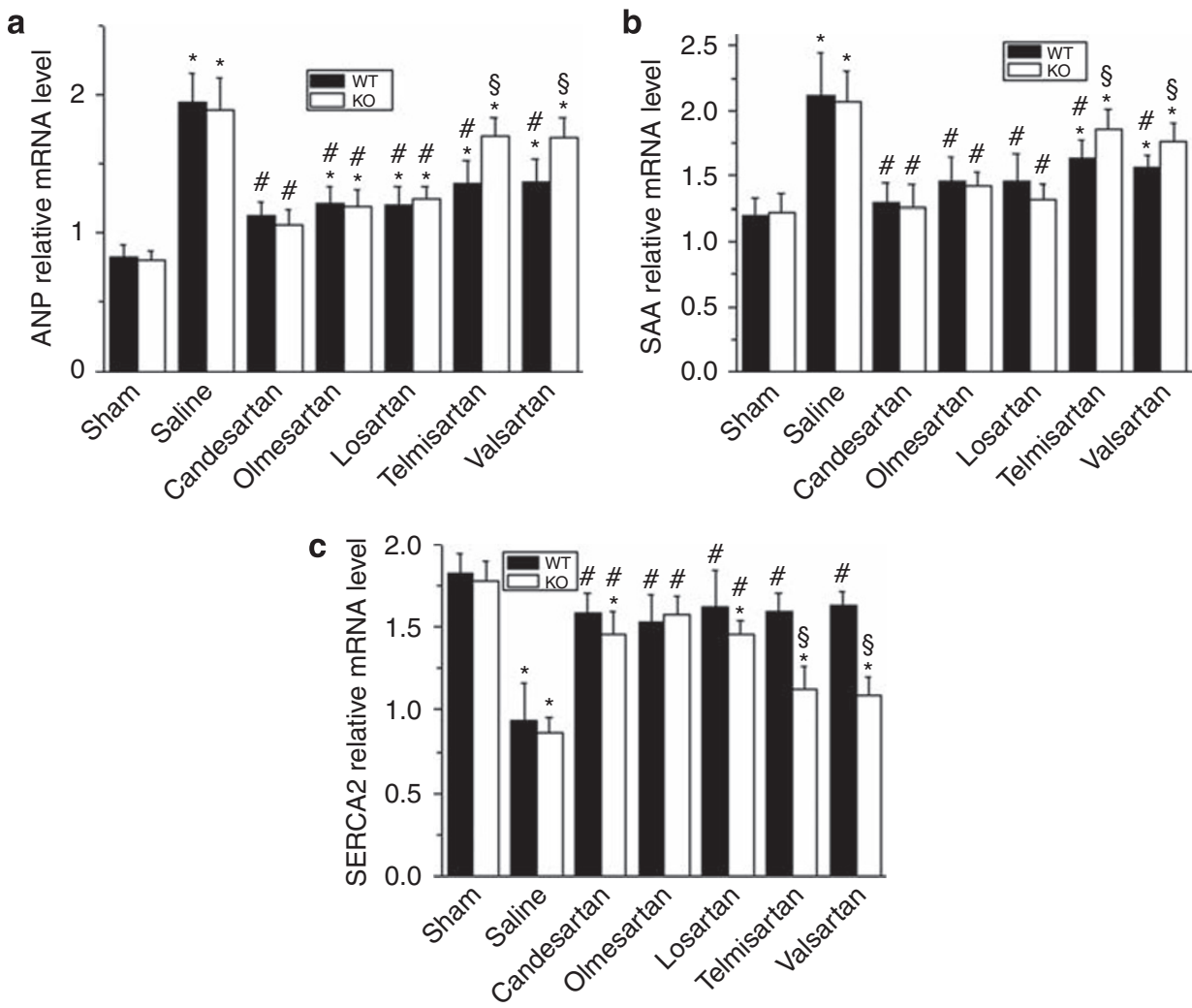

Figure 6 Reprogramming of specific gene expressions in the hearts. (a-c) Quantifications of ANP, SAA and SERCA2 gene expressions detected by real-time PCR. ${ }^{*} P<0.05$ vs. sham-operated mice of the same genotype; ${ }^{\#} P<0.05$ vs. saline-treated TAC mice of the same genotype; ${ }^{\circledR} P<0.05$ vs. same ARB-treated WT mice with TAC. 
can explain at least in part the in vivo efficacy of Losartan in the WT and the ATG KO mice.

As indicated by our results, cardiac hypertrophy induced by pressure overload in WT mice was also significantly reduced by Telmisartan and Valsartan. However, the degree of reduction tended to be less by Telmisartan and Valsartan, which lack activities of the inverse agonist, than those by Olmesartan, Candesartan and Losartan, which act as inverse agonists, although the difference between them was not significant. It has been known that AngII and AT1 receptor have a critical role in pressure overload-induced cardiac hypertrophy. Pressure overload induces cardiac hypertrophy in WT mice mainly through activation of AT1 receptor both by mechanical stress and by AngII. Although Telmisartan and Valsartan could not block the activation of AT1 receptor induced by mechanical stress, they did block the activation of AT1 receptor induced by AngII. This may explain why the hypertrophy induced by pressure overload in WT mice could be effectively reduced by Telmisartan and Valsartan but the degree of reduction tended to be less than that by Olmesartan, Candesartan and Losartan. In the ATG KO mice, however, as pressure overload induces cardiac hypertrophy through activation of AT1 receptor only by mechanical stress, Telmisartan and Valsartan could not exert inhibitory effects.

Our study demonstrated that some ARBs such as Candesartan, Olmesartan and Losartan strongly inhibit pressure overload-induced cardiac hypertrophy in both the presence and absence of AngII. The insurmountability and inverse agonism against the AT1-R are considered to be crucial for the efficacy.

Some of our present data are different from the results of some clinical trials regarding heart failure (VALHEFT, Charm-added). Although our experimental data showed a less significant regression of pressure overload-induced cardiac hypertrophy by Valsartan and Telmisartan than by Olmesartan, Candesartan and Losartan, data from clinical trials including VALHEFT and ONTARGET revealed that Valsartan and Telmisartan can markedly decrease the cardiovascular death or hospital admission of patients with chronic heart failure. Also, some other clinical trials such as LIFE, CHARM-added and $\mathrm{COACH}$ studies revealed significant cardioprotection of ARBs in patients with hypertension or chronic heart failure. Although we don't know the exact reason for these differences, there are two possibilities. First, we used the TAC mice model to produce pressure overload and compared the regressive effects of ARBs on cardiac hypertrophy at high blood pressure, which is different from those observed in patients whose blood pressure was effectively controlled. Second, we observed the inhibitory effects of different ARBs on cardiac hypertrophy at 2 weeks after pressure overload, a stage of compensatory hypertrophy without heart failure during which the effect of ARBs on heart failure was not involved, which is also different from other clinical trials. The different effects of ARBs on cardiac protection should be tested in hypertensive animals in a similar condition to that of clinics and should be observed for a sufficient period in future studies. As there is still a shortage of authoritative clinical studies to compare the differences in cardiac protection provided by all commonly prescribed ARBs in clinics, we do not know at present whether there would be differences in cardiac protection against chronic heart failure among different ARBs in patients. However, we believe that our animal study will provide helpful references for future clinical trials and directive information for the clinical choice among various ARBs.

The efficacies of ARBs on cardiac protection during hypertension are different, which are associated with the differences in chemical structures of the drugs. Elucidation of the molecular interaction model for the chemical structure of ARB and AT1-R will be helpful to discriminate the ARBs according to the efficacies in AT1-R inactivation, and to develop a novel ARB with superb efficacy not only in blood pressure lowering but also in organ protection. In addition, as our study only observed the efficacies of ARBs for 2 weeks after TAC, it remains to be investigated whether inverse agonists prevent cardiac damage more effectively than competitive antagonists for a long term.

\section{CONFLICT OF INTEREST}

The authors declare no conflict of interest.

\section{ACKNOWLEDGEMENTS}

We thank Jianguo Jia, Guoping Zhang and Jingzhen Zhu for their excellent technical assistance. This work was supported by the grants from National Science Fund for Distinguished Young Scholars from the National Natural Science Foundation of China (30525018), National Basic Research Program of China (2007CB512003), National Natural Science Foundation of China (No. 30670840), Science and Technology Commission of Shanghai Municipality (05XD14003) and China Doctoral Foundation (20060246079).

1 Levy D, Garrison RJ, Savage DD, Kannel WB, Castelli WP. Prognostic implications of echocardiographically determined left-ventricular mass in the FraminghamHeart-Study. N Engl J Med 1990; 322: 1561-1566.

2 Frohlich ED, Apstein C, Chobanian AV, Devereux RB, Dustan HP, Dzau V, Fauadtarazi F, Horan MJ, Marcus M, Massie B, Pfeffer MA, Re RN, Roccella EJ, Savage D, Shub C. Medical progress-the heart in hypertension. N Engl J Med 1992; 327: 998-1008.

3 Levy D, Larson MG, Vasan RS, Kannel WB, Ho KKL. The progression from hypertension to congestive heart failure. J Am Med Assoc 1996; 275: 1557-1562.

4 Yamazaki T, Komuro I, Kudoh S, Zou YZ, Shiojima I, Mizuno T, Takano H, Hiroi Y, Ueki K, Tobe K, Kadowaki T, Nagai R, Yazaki Y. Angiotensin-II partly mediates mechanical stress-induced cardiac-hypertrophy. Circ Res 1995; 77: 258-265.

5 Sadoshima J, Xu YH, Slayter HS, Izumo S. Autocrine release of angiotensin-II mediates stretch-induced hypertrophy of cardiac myocytes in-vitro. Cell 1993; 75: 977-984.

6 Zaman MA, Oparil S, Calhoun DA. Drugs targeting the renin-angiotensin-aldosterone system. Nat Rev Drug Discov 2002; 1: 621-636.

7 Kjeldsen SE, Dahlof B, Devereux RB, Julius S, Aurup P, Edelman J, Beevers G, de Faire U, Fyhrquist F, Ibsen H, Kristianson K, Lederballe-Pedersen O, Lindholm LH, Nieminen MS, Omvik P, Oparil S, Snapinn S, Wedel H, Grp LS. Effects of losartan on cardiovascular morbidity and mortality in patients with isolated systolic hypertension and left ventricular hypertrophy-A Losartan Intervention For Endpoint Reduction (LIFE) substudy. J Am Med Assoc 2002; 288: 1491-1498.

8 Okin PM, Devereux RB, Jern S, Kjeldsen SE, Julius S, Nieminen MS, Snapinn S, Harris KE, Aurup P, Edelman JM, Dahlof B, Investigators LS. Regression of electrocardiographic left ventricular hypertrophy by Losartan versus atenolol-The Losartan Intervention for Endpoint Reduction in Hypertension (LIFE) Study. Circulation 2003; 108: 684-690.

9 O'Keefe JH, Wetzel M, Moe RR, Brosnahan K, Lavie CJ. Should an angiotensinconverting enzyme inhibitor be standard therapy for patients with atherosclerotic disease? J Am Coll Cardiol 2001; 37: 1-8.

10 de Gasparo M, Catt KJ, Inagami T, Wright JW, Unger T. International Union of Pharmacology. XXIII. The angiotensin II receptors. Pharmacol Rev 2000; 52: 415-472.

11 Zou YZ, Akazawa H, Qin YJ, Sano M, Takano H, Minamino T, Makita N, Iwanaga K, Zhu WD, Kudoh S, Toko H, Tamura K, Kihara M, Nagai T, Fukamizu A, Umemura S, liri T, Fujita T, Komuro I. Mechanical stress activates angiotensin II type 1 receptor without the involvement of angiotensin II. Nat Cell Biol 2004; 6: 499-506.

12 Qin Y, Yasuda N, Akazawa H, Ito K, Kudo Y, Liao CH, Yamamoto R, Miura SI, Saku K, Komuro I. Multivalent ligand-receptor interactions elicit inverse agonist activity of AT(1) receptor blockers against stretch-induced AT(1) receptor activation. Hypertens Res 2009; 32: 875-883.

13 Oparil S. Newly emerging pharmacologic differences in angiotensin II receptor blockers. Am J Hypertens 2000; 13: 18S-24S.

14 Morsing P, Adler G, Brandt-Eliasson U, Karp L, Ohlson K, Renberg L, Sjoquist PO, Abrahamsson T. Mechanistic differences of various AT(1)-receptor blockers in isolated vessels of different origin. Hypertension 1999; 33: 1406-1413.

15 Tanimoto K, Sugiyama F, Goto Y, Ishida J, Takimoto E, Yagami K, Fukamizu A, Murakami K. Angiotensinogen-deficient mice with hypotension. J Biol Chem 1994; 269: 31334-31337.

16 Rockman HA, Ross RS, Harris AN, Knowlton KU, Steinhelper ME, Field LJ, Ross J, Chein KR. Segregation of atrial-specific and inducible expression of an atrial-natriuretic-factor transgene in an in vivo murine model of cardiac-hypertrophy. Proc Natl Acad Sci USA 1991; 88: 8277-8281. 
17 Liao YL, Ishikura F, Beppu S, Asakura M, Takashima S, Asanuma H, Sanada S, Kim J, Ogita H, Kuzuya T, Node K, Kitakaze M, Hori M. Echocardiographic assessment of LV hypertrophy and function in aortic-banded mice: necropsy validation. Am J Physiol Heart Circul Physiol 2002; 282: H1703-H1708.

18 Moens AL, Takimoto E, Tocchetti CG, Chakir K, Bedja D, Cormaci G, Ketner EA, Majmudar M, Gabrielson K, Halushka MK, Mitchell JB, Biswal S, Channon KM, Wolin MS, Alp NJ, Paolocci N, Champion HC, Kass DA. Reversal of cardiac hypertrophy and fibrosis from pressure overload by tetrahydrobiopterin - efficacy of recoupling nitric oxide synthase as a therapeutic strategy. Circulation 2008; 117: 2626-2636.

19 Liao YL, Asakura M, Takashima S, Ogai A, Asano Y, Asanuma H, Minamino T, Tomoike H, Hori M, Kitakaze M. Benidipine, a long-acting calcium channel blocker, inhibits cardiac remodeling in pressure-overloaded mice. Cardiovasc Res 2005; 65: 879-888.

20 Komuro I, Yazaki Y. Control of cardiac gene-expression by mechanical-stress. Annu Rev Physiol 1993; 55: 55-75.
21 Zou YZ, Hiroi Y, Uozumi H, Takimoto E, Toko H, Zhu WD, Kudoh S, Mizukami M, Shimoyama M, Shibasaki F, Nagai R, Yazaki Y, Komuro I. Calcineurin plays a critical role in the development of pressure overload-induced cardiac hypertrophy. Circulation 2001; 104: 97-101.

22 Van Liefde I, Vauquelin G. Sartan-AT(1) receptor interactions: in vitro evidence for insurmountable antagonism and inverse agonism. Mol Cell Endocrinol 2009; 302: 237-243.

23 Le MT, Pugsley MK, Vauquelin G, Van Liefde I. Molecular characterisation of the interactions between olmesartan and telmisartan and the human angiotensin II AT(1) receptor. Br J Pharmacol 2007; 151: 952-962.

24 Akazawa H, Yasuda N, Komuro I. Mechanisms and functions of agonist-independent activation in the angiotensin II type 1 receptor. Mol Cell Endocrinol 2009; 302: 140-147.

25 Milligan G. Constitutive activity and inverse agonists of $G$ protein-coupled receptors: a current perspective. Mol Pharmacol 2003; 64: 1271-1276.

Supplementary Information accompanies the paper on Hypertension Research website (http://www.nature.com/hr) 